\title{
Experimental and Numerical Study on Combustion Mechanism of Liquid Fuel Spray Entering Gaseous Flame Front*
}

\author{
Mariko NAKAMURA**, Fumiteru AKAMATSU ${ }^{* * *}$, \\ Ryoichi KUROSE**** and Masashi KATSUKI** $^{* *}$
}

\begin{abstract}
Experimental observations and numerical simulations are conducted on combustion processes of n-decane polydisperse spray entering a gaseous flat flame stabilized in a laminar 2D counterflow configuration. For the gaseous phase, Eulerian mass, momentum, energy, and species conservation equations are solved. For the disperse phase, all individual droplets are tracked without using a droplet parcel model. The experimental results show that blue flames and luminous flames are observed and there are unsteady changes in the behavior. The numerical results show that the spray flame structures vary depending on the supplied quantities of liquid fuel spray. Furthermore, the instantaneous flame structures are consistent with the typical flame structures observed with the experiment.
\end{abstract}

Key Words: Combustion, Liquid Fuel, Spray Flame, Droplet, Laminar Counterflow

\section{Introduction}

Spray flames are complicated reacting turbulent phenomena in which fuel atomization, droplet dispersion into gaseous phase, evaporation, mixing of fuel vapor with air, etc., simultaneously occur and interact with each other. Therefore, the underlying physics governing these processes has not been well understood.

In recent years, we have investigated spray flames in a laminar counterflow and turbulent jet using advanced laser-based diagnostics with high temporal and spatial resolution. The experiments showed that the spatial nonuniformity of the spray tends to form groups of droplets ${ }^{(1)}$, and the temporal and spatial variations of their behavior cause a change in the flame structure ${ }^{(2),(3)}$. Furthermore, the spray flame structure differs depending on the conditions of liquid fuel supply and spray characteristics ${ }^{(4),(5)}$. However, since it is presently impossible to experimen-

* Received 1st February, 2006 (No. 04-0729). Japanese Original: Trans. Jpn. Soc. Mech. Eng., Vol.71, No.707, B (2005), pp.1921-1928 (Received 21st June, 2004)

** Kobelco Eco-Solutions CO., LTD., 1-1-4 Murotani, Nishi-ku, Kobe, Hyogo 651-2241, Japan.

E-mail: m.nakamura@kobelco-eco.co.jp

*** Department of Mechanical Engineering, Osaka University, 2-1 Yamadaoka, Suita, Osaka 565-0871, Japan

**** Central Research Institute of Electric Power Industry (CRIEPI), 2-6-1 Nagasaka, Yokosuka, Kanagawa 2400196, Japan tally measure all physical quantities in spray flames, a detailed mechanism has not been clarified yet. Accordingly, experiments are conducted and numerical simulations are performed simultaneously to elucidate the underlying physics of spray combustion.

In this paper, time-dependent numerical simulations are carried out on the combustion processes of n-decane polydisperse spray entering a gaseous flat flame stabilized in a laminar 2D counterflow configuration. The results are compared with the corresponding experimental results in order to clarify the combustion mechanism of droplets and droplet clusters as well as the variation of the spray flame structure caused by the non-uniformity of the physical quantities of the spray. In order to observe the spray flame structures in detail, an appropriate flowfield where advanced optical techniques can be used should be chosen to extract the basic characteristics of spray flames. The spray flames stabilized in a counterflow have been accepted as a useful tool for the investigation of their structures $^{(6)-(13)}$, because the similarity solution ${ }^{(7)}$ on the centerline defines the entire flowfield if a monodisperse smallsized spray of highly volatile liquid fuel is used and almost all droplets prevaporize before entering the reaction zone. However, the numerical simulation with the similarity solution becomes inaccurate if we deal with actual polydisperse spray flames in which the combustion reaction zone interacts intensively with the unburned spray. Since the present study deals with an n-decane polydis- 
perse spray (liquid fuel with a relatively low volatility) including large droplets, an original numerical code for the two-dimensional counterflow field ${ }^{(14),(15)}$ is adopted instead of the code with the similarity solution. In the numerical code, Eulerian mass, momentum, energy, and species conservation equations are solved for the gaseous phase. With respect to the disperse phase, all individual droplets are tracked in a Lagrangian manner without using a droplet parcel model, in which parcels (imaginary particle containing many fuel droplets) are tracked to reduce the calculation load for tracking many droplets in the flowfield.

\section{Nomenclature}

$d_{l}:$ droplet diameter

$h:$ specific total enthalpy

$m_{l}:$ droplet mass $\left(=(\pi / 6) \cdot d_{l}^{3} \rho_{l}\right)$

$\dot{m}_{l}$ : droplet evaporation rate

$Q_{l}$ : convective heat transfer

$S_{\text {combu,k }}$ : source term of the $k$-th species with combustion

$S_{l}$ : source term due to interactions between the gaseous phase and droplets

$T$ : gaseous temperature

$T_{l}:$ droplet temperature

$\boldsymbol{V}:=(u, v)$ velocity vector of gaseous phase

$V_{l}:=\left(u_{l}, v_{l}\right)$ velocity vector of droplet

$Y_{k}$ : mass fraction of the $k$-th species

\section{Greek Symbols}

$$
\begin{aligned}
\rho & : \text { gaseous density } \\
\rho_{l} & : \text { droplet density } \\
\phi & : \text { gaseous phase equivalence ratio }
\end{aligned}
$$

\section{Experimental Apparatus}

Figure 1 shows a photograph of the experimental burner and structure of the burner port. The upstream part of the upper burner port has a co-axial pipe structure and the diameter of the internal pipe is $30 \mathrm{~mm}$. The liquid fuel spray atomized in the chamber above the burner passes

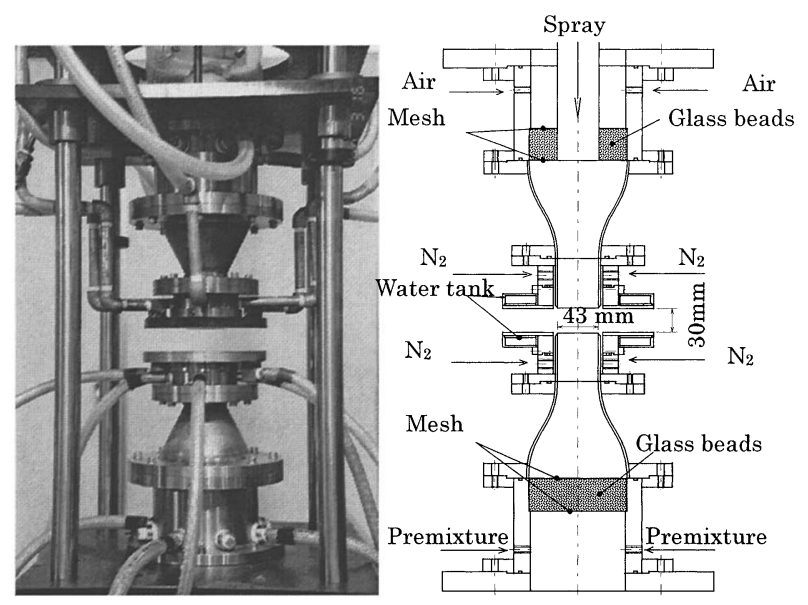

Fig. 1 Experimental apparatus through an internal pipe. The main air stream of the upper port is supplied from four directions from outside the co-axial pipe, rectified using glass beads, and mixed with the liquid fuel spray to form premixed spray steam. This steam passes through a contraction nozzle, and it is supplied from the upper burner port (diameter: $43 \mathrm{~mm}$ ) with a uniform vertical velocity. The main air stream of the lower side is mixed with gaseous fuel (city gas 13 A) to form a premixture. The premixture is rectified using glass beads, passed through a contraction nozzle, and supplied from the lower burner port (diameter: $43 \mathrm{~mm}$ ) with a uniform vertical velocity. For both the upper and lower ports, co-axial nitrogen stream with the same velocity as that of the main stream is issued from the outermost annular port (i.d. of $43 \mathrm{~mm}$ and o.d. of $47 \mathrm{~mm}$ ) to suppress shear with the atmosphere and prevent the flame from being attached to the burner limb. In addition, a water-cooled disk (diameter: $160 \mathrm{~mm}$ ) is equipped in the vicinity of each burner port to protect the flame from atmospheric disturbances.

The supplied liquid fuel (n-decane: $\mathrm{C}_{10} \mathrm{H}_{22}$ ) is pressurized by the fuel pump and atomized by a pressure nozzle in the chamber located above the burner. A shutter is equipped in the lower part of the chamber in order to start fuel spray injection at any instant.

In the present experiment, the separation between the upper and lower ports is set at $30 \mathrm{~mm}$. The gaseous velocity from both ports is $0.6 \mathrm{~m} / \mathrm{s}$. The equivalence ratio of the premixture from the lower port is 0.6. The flat flame is stabilized in a laminar counterflow where the stretch ratio is $401 / \mathrm{s}$. When the shutter is opened, polydisperse ndecane spray is supplied to the high temperature region generated by the flat flame to form the spray flame. The time-averaged bulk equivalence ratio of the spray is 0.42 .

\section{Calculation Method}

The planar two-dimensional flowfield for the calculation is shown in Fig. 2. The origin of the calculation domain is located at the center of the upper burner port. The gaseous species considered in the calculations are $\mathrm{O}_{2}$, $\mathrm{N}_{2}, \mathrm{CO}_{2}, \mathrm{H}_{2} \mathrm{O}$, and $\mathrm{C}_{10} \mathrm{H}_{22}$. Their transport properties and thermodynamic data are obtained from $\operatorname{CHEMKIN}^{(16),(17)}$. In order to consider the temperature dependence of the

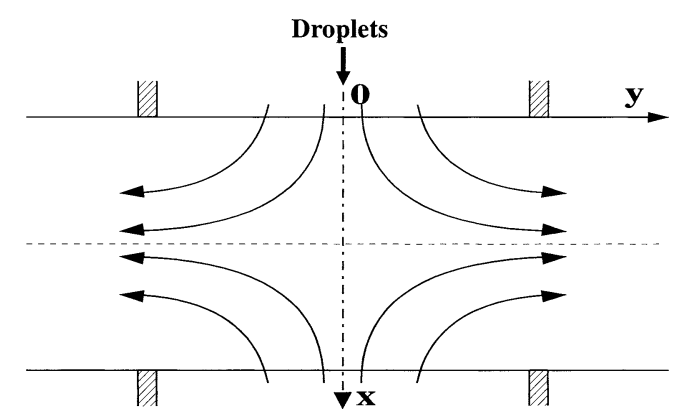

Fig. 2 Schematic of the flowfield 
properties of liquid n-decane, the Gunn-Yamada equation for density, the Sato-Riedel equation for heat conductivity, and the Sterning-Brown equation for specific heat are used, respectively ${ }^{(18)}$. The governing equations considered for the gaseous phase are those for mass, momentum, energy, and species mass conservation, given as follows:

$$
\begin{aligned}
& \frac{\partial \rho}{\partial t}+\frac{\partial \rho u}{\partial x}+\frac{\partial \rho v}{\partial y}=S_{l, m} \\
& \frac{\partial(\rho u)}{\partial t}+\frac{\partial}{\partial x}\left(\rho u u-\mu \frac{\partial u}{\partial x}\right)+\frac{\partial}{\partial y}\left(\rho v u-\mu \frac{\partial u}{\partial y}\right) \\
& =-\frac{\partial P}{\partial x}+\frac{\partial}{\partial x}\left(\mu \frac{\partial u}{\partial x}\right)+\frac{\partial}{\partial y}\left(\mu \frac{\partial v}{\partial x}\right) \\
& \quad-\frac{2}{3} \frac{\partial}{\partial x}\left[\mu\left(\frac{\partial u}{\partial x}+\frac{\partial v}{\partial y}\right)\right]+\rho g+S_{l, u} \\
& \frac{\partial(\rho v)}{\partial t}+\frac{\partial}{\partial x}\left(\rho u v-\mu \frac{\partial v}{\partial x}\right)+\frac{\partial}{\partial y}\left(\rho v v-\mu \frac{\partial v}{\partial y}\right) \\
& =-\frac{\partial P}{\partial y}+\frac{\partial}{\partial x}\left(\mu \frac{\partial u}{\partial y}\right)+\frac{\partial}{\partial y}\left(\mu \frac{\partial v}{\partial y}\right) \\
& \quad-\frac{2}{3} \frac{\partial}{\partial y}\left[\mu\left(\frac{\partial u}{\partial x}+\frac{\partial v}{\partial y}\right)\right]+S_{l, v} \\
& \frac{\partial(\rho h)}{\partial t}+\frac{\partial}{\partial x}\left(\rho u h-\rho a \frac{\partial h}{\partial x}\right)+\frac{\partial}{\partial y}\left(\rho v h-\rho a \frac{\partial h}{\partial y}\right) \\
& =-\frac{\partial}{\partial x}\left(\sum_{k=1}^{n} h_{k}\left(\rho a-\rho D_{k}\right) \frac{\partial Y_{k}}{\partial x}\right) \\
& \quad-\frac{\partial}{\partial y}\left(\sum_{k=1}^{n} h_{k}\left(\rho a-\rho D_{k}\right) \frac{\partial Y_{k}}{\partial y}\right)+S_{l, h} \\
& \frac{\partial\left(\rho Y_{k}\right)}{\partial t}+\frac{\partial}{\partial x}\left(\rho u Y_{k}-\rho D_{k} \frac{\partial Y_{k}}{\partial x}\right)+\frac{\partial}{\partial y}\left(\rho v Y_{k}-\rho D_{k} \frac{\partial Y_{k}}{\partial y}\right) \\
& =S_{c o m b u, k}+S_{l, Y_{k}},
\end{aligned}
$$

where $\rho$ is the gaseous phase density; $u$ and $v$, the gaseous phase velocity in the $x$ and $y$ directions, respectively; $\mu$, the gaseous viscosity; $P$, the static pressure; $g$, the gravitational force; $h$, the specific total enthalpy; $a$, the gaseous thermal diffusivity; and $h_{k}, Y_{k}$, and $D_{k}$ are the specific enthalpy, mass fraction, and mass diffusion coefficient of the $k$-th species, respectively. The source term $S_{c o m b u, k}$ is due to combustion. The source terms $S_{l}$ represent the interactions between the gaseous and disperse phases. The gaseous phase density $\rho$ is calculated from the equation of a state for ideal gas. These governing equations are solved by a finite volume method using a SIMPLE algorithm ${ }^{(19)}$. The spatial integration is approximated by a fourth-order central difference scheme, and the time integration is performed via a fully implicit method.

Mass, heat, and momentum interactions between the gaseous and disperse phases are evaluated on the basis of a PSI-Cell model ${ }^{(20)}$. The interaction terms between the phases during a calculation time step are considered at the final droplet location of the time step.

The equation describing a droplet motion is expressed as follows, provided that there is no collision and no breakup of droplets:

$$
m_{l} \frac{d \boldsymbol{V}_{l}}{d t}=\boldsymbol{F}+m_{l} \cdot \boldsymbol{g}
$$

where $\boldsymbol{F}=\left(F_{x}, F_{y}\right)$ is the drag force acting on a droplet by the gaseous phase expressed as follows:

$$
\boldsymbol{F}=\frac{1}{8} \cdot \pi d_{l}^{2} \rho\left(\boldsymbol{V}-\boldsymbol{V}_{l}\right)\left|\boldsymbol{V}-\boldsymbol{V}_{l}\right| C_{D},
$$

where $m_{l}$ is the droplet mass given by $m_{l}=(\pi / 6) \cdot d_{l}^{3} \rho_{l}$; $\rho_{l}$, the droplet density; $\boldsymbol{V}=(u, v)$, the velocity vector of the gaseous phase; $\boldsymbol{V}_{l}=\left(u_{l}, v_{l}\right)$, the velocity vector of the droplet; and $\boldsymbol{g}=(g, 0)$, the vector of the gravitational force. The drag coefficient of a droplet $C_{D}$ proposed by Kurose et al. ${ }^{(21)}$ is adopted.

$$
\begin{aligned}
& C_{D}=\frac{24}{R e_{d}} \cdot\left(\frac{1+0.0545 R e_{d}+0.1 R e_{d}^{1 / 2}\left(1-0.03 R e_{d}\right)}{1+b\left|R e_{n}\right|^{c}}\right), \\
& b=0.06+0.077 \exp \left(-0.4 R e_{d}\right), \\
& c=0.4+0.77 \exp \left(-0.04 R e_{d}\right), \\
& R e_{d}=\frac{\rho\left|\boldsymbol{V}-\boldsymbol{V}_{l}\right| d_{l}}{\mu}
\end{aligned}
$$

where $R e_{n}$ is given by $R e_{n}=d_{l} V_{n}^{*} / v ; V_{n}^{*}$, the blowing velocity; and $v$, the gaseous kinematic viscosity.

With respect to the droplet evaporation model, a nonequilibrium Langmuir-Knudsen model is chosen ${ }^{(22),(23)}$. Based on this model, a temporal change in the mass, temperature, and diameter of a droplet is expressed as follows:

$$
\begin{aligned}
& \dot{m}_{l}=\frac{d m_{l}}{d t}=-\pi d_{l} \rho D_{F} S h \cdot \ln \left(1+B_{M}\right), \\
& \begin{aligned}
\frac{d T_{l}}{d t} & =\frac{Q_{l}+\dot{m}_{l} L\left(T_{l}\right)}{m_{l} c_{p l}} \\
& =\frac{N u}{3 \operatorname{Pr}}\left(\frac{c_{p}}{c_{p l}}\right)\left(\frac{f}{\tau_{l}}\right)\left(T-T_{l}\right)+\left(\frac{\dot{m}_{l}}{m_{l}}\right) \frac{L\left(T_{l}\right)}{c_{p l}},
\end{aligned} \\
& \frac{d\left(d_{l}\right)^{3}}{d t}=-\frac{6 \dot{m}_{l}}{\pi \rho_{l}},
\end{aligned}
$$

where $D_{F}$ is the mass diffusion coefficient of the fuel; Sh, the Sherwood number ${ }^{(22),(23)} ; B_{M}$, the mass transfer number ${ }^{(22),(23) ;} Q_{l}$, the convective heat transfer; $L\left(T_{l}\right)$, the latent heat at the droplet temperature $T_{l} ; c_{p}$ and $c_{p l}$, the specific heat of the mixture and liquid fuel at constant pressure, respectively; $\mathrm{Nu}$, the Nusselt number ${ }^{(22),(23)} ; \mathrm{Pr}$, the Prandtl number; $f$, the correction function of heat transfer for the evaporation droplet ${ }^{(22),(23)} ; \tau_{l}$, the particle response time defined by $\tau_{l}=\rho_{l} d_{l}^{2} / 18 \mu ; T_{l}$, the droplet temperature; and $T$, the gaseous phase temperature. The equations for the droplets are integrated using a second-order AdamsBashforth method.

The source terms $S_{l}$, due to interactions between gaseous and disperse phases, are expressed using the total number of droplets $N$ distributing the source term to the control volume in the gaseous phase calculation. For the equation of mass conservation, 


$$
S_{l, m}=-\sum_{N} \frac{\dot{m}_{l}}{\Delta V}
$$

For the equations of momentum conservation,

$$
\begin{aligned}
& S_{l, u}=-\sum_{N} \frac{F_{x}+\dot{m}_{l} u_{l}}{\Delta V}, \\
& S_{l, v}=-\sum_{N} \frac{F_{y}+\dot{m}_{l} v_{l}}{\Delta V} .
\end{aligned}
$$

For the equation of energy conservation,

$$
S_{l, h}=-\left(\sum_{N}\left[\frac{1}{2} \frac{d}{d t}\left(m_{l} \boldsymbol{V}_{l} \cdot \boldsymbol{V}_{l}\right)+Q_{l}+\dot{m}_{l} h_{F, l}\right]\right) / \Delta V .
$$

For the equations of species conservation,

$$
S_{l, Y_{F}}=-\sum_{N} \frac{\dot{m}_{l}}{\Delta V},
$$

where $\Delta V$ is the volume of the control volume in the gaseous phase calculation and $h_{F, l}$ is the evaporated vapor enthalpy.

For the combustion reaction model, a single-step global reaction of n-decane ${ }^{(24)}$ is adopted. The source term $S_{c o m b u, k}$, in the equations of species conservation, is expressed using the combustion reaction rate per unit volume $R_{F}$, as follows:

$$
S_{c o m b u, k}=-\frac{n_{k}}{n_{F}} \cdot \frac{W_{k}}{W_{F}} \cdot R_{F},
$$

where $n_{k}$ and $n_{F}$ are the molar stoichiometric coefficients of the $k$-th species and fuel, respectively, of the single-step global reaction (positive for the production side); and $W_{k}$ and $W_{F}$ are the molecular weights of the $k$-th species and fuel, respectively.

The calculation domain $(0 \leq x \leq 20 \mathrm{~mm}, 0 \leq y \leq$ $40 \mathrm{~mm}$ ) is divided into $200 \times 400$ equally spaced computational grids in the $x$ and $y$ directions, respectively, which generate an actual control volume size of $100 \mu \mathrm{m}$ $\times 100 \mu \mathrm{m}$. The calculation time step for the gaseous phase is set at $0.1 \mathrm{~ms}$. The calculation time step for droplets $\Delta t_{l}$ is decided to satisfy the condition $\Delta t_{l} / \tau<0.001$, where $\tau$ is the heat relaxation time denoted as $\tau=3 \operatorname{Prc}_{p l} \tau_{l} / N u c_{p} f$.

Atmospheric air $(T=300 \mathrm{~K}$, oxygen mass fraction $Y_{O 2}=0.2357$ ) is issued from the upper port in a range of $-10 \mathrm{~mm} \leq y \leq 10 \mathrm{~mm}$. From the lower port, the premixture $(\phi=0.6)$ is issued in a range of $-3 \mathrm{~mm} \leq y \leq 3 \mathrm{~mm}$, and atmospheric air is issued in ranges of $-10 \mathrm{~mm} \leq y<-3 \mathrm{~mm}$ and $3 \mathrm{~mm}<y \leq 10 \mathrm{~mm}$. The initial gaseous velocity is $0.4 \mathrm{~m} / \mathrm{s}$ and the global stretch ratio of the counterflow is $40 \mathrm{~s}^{-1}$. A polydisperse $\mathrm{n}$-decane spray is injected from the upper port in the range of $-3 \leq y \leq 3 \mathrm{~mm}$ at a velocity of $0.4 \mathrm{~m} / \mathrm{s}$. The droplet size distribution obtained by the PDA measurement is used in the calculations (Fig. 3). A uniform stochastic process determines the initial position of each droplet. The initial condition is the gaseous flat flame formed by the premixture from the lower port. Spray injection starts at $t=0 \mathrm{~ms}$ and the calculations are conducted until $t=200 \mathrm{~ms}(0.1 \mathrm{~ms} \times 2000$ time steps $)$.

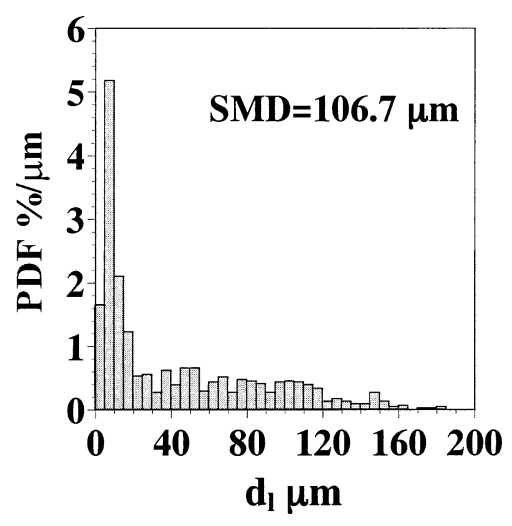

Fig. 3 Droplet size distribution

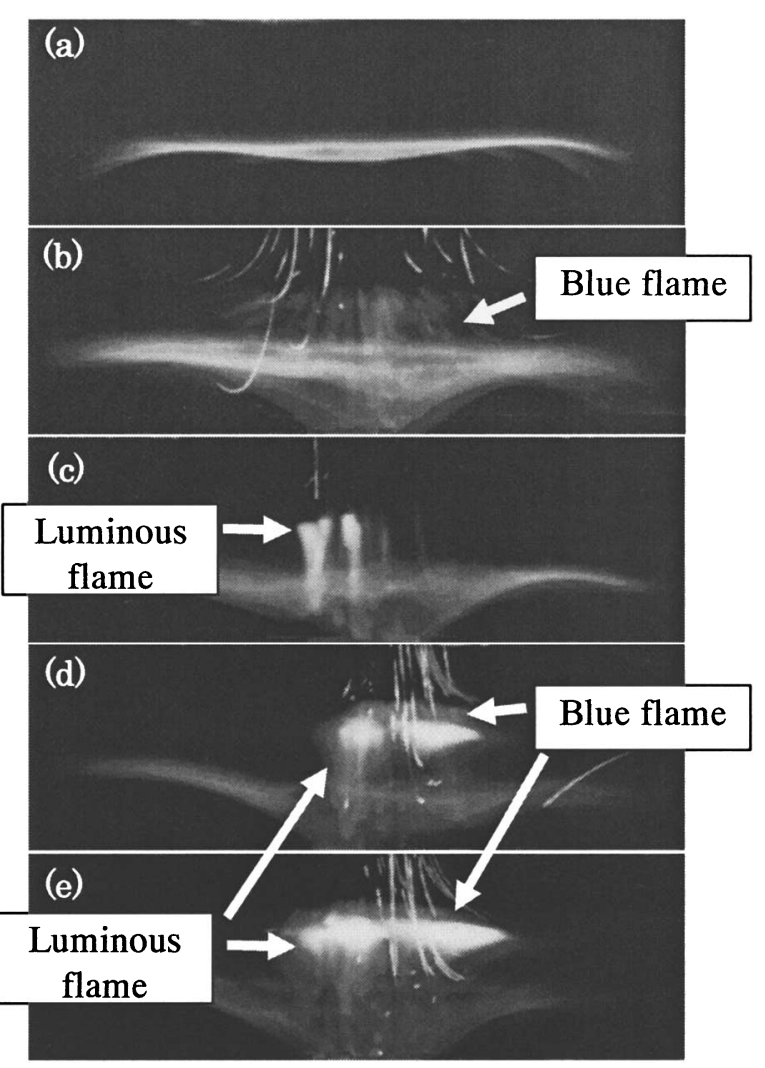

Fig. 4 Photographs of spray flames

\section{Results and Discussion}

\section{1 Experimental Results}

In order to observe the shapes of the spray flame, the photographs are taken using a film camera (exposure time is $1 / 15 \mathrm{~s}$ ). Figures $4(\mathrm{a})-(\mathrm{e})$ show the photographs of the gaseous flame before the injection of the spray and its variation with spray addition. The height of these photographs is $30 \mathrm{~mm}$ and corresponds to the separation between the upper and lower ports. These photographs show the typical flame appearance after spray addition, but have not been taken in time-series.

Figure 4 (a) shows the flat flame of the gaseous fuel, which is stabilized at $h=18 \mathrm{~mm}$ from the upper port, 
before spray addition. Since the gaseous velocity at the burner port is almost uniform, the flame is stable without any movement.

Then, the spray is supplied from the airflow side toward the gaseous flat flame. Large droplets are visualized with a light sheet of an $\mathrm{Ar}$ ion laser (the wavelength is $524.5 \mathrm{~nm}$ ) illuminating the vertical section including the center axis of the burner.

Figure 4 (b) depicts the blue flames in the high temperature region of the burned gases when the droplets are supplied uniformly and stably. The larger droplets penetrate the gaseous flame of the premixture supplied from the lower port and are pushed back by the flow from the lower port. Figure 4 (c) shows the luminous flames that appear intermittently.

If a large amount of spray is supplied in a short time, a luminous flame lump accompanied by a thin blue flame in the upper portion is observed, as shown in Fig. 4(d). Furthermore, the shape of the luminous flame lump varies unsteadily depending on the fluctuation of the supplied quantity of the liquid fuel or the characteristics of the spray (see Fig. $4(\mathrm{e}))$.

\subsection{Numerical Results}

The spray flame structure obtained by the experiment is discussed in detail using numerical simulations. For the simulations, three cases of the bulk equivalence ratio of the spray, $\phi_{l}=0.21,0.42$ (the same as the experiment), and 1.26, are assumed because the amount of the supplied spray fluctuates in the experiment due to the characteristics of the atomization process.

Figure 5 shows the time-averaged axial ( $x$ direction) profiles of (a) gaseous velocity in the axial direction $(y=$ $0 \mathrm{~mm}) u$, (b) the gaseous temperature $T$, (c) the mass fraction of oxygen $Y_{O 2}$, and (d) the mass fraction of n-decane, $Y_{\mathrm{C} 1 \mathrm{OH} 22}$. These results are the time-averaged properties in the quasi-steady state between $t=100 \mathrm{~ms}$ and $200 \mathrm{~ms}$ from the initiation of the spray addition.

In Fig. 5 (a), due to the spray addition, $u$ increases on the spray flow side of the stagnation surface, and the increase in velocity when $\phi_{l}=1.26$ is the maximum in the three cases. In Fig. 5 (b), when $\phi_{l}=0.21$, the gaseous temperature $T$ is lower than that in the other two cases in the entire region; the maximum temperature indicates a value lower than the other two cases by approximately $500 \mathrm{~K}$.

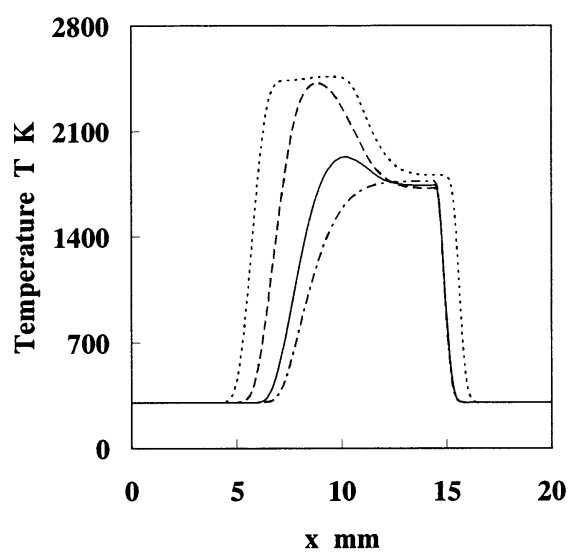

(b) Temperature

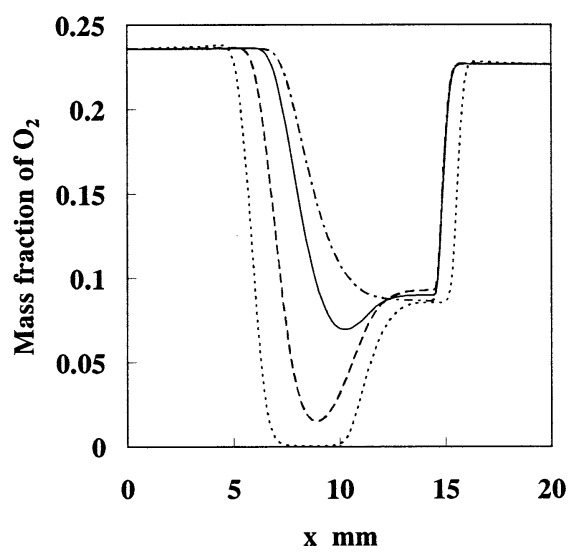

(c) Mass fraction of $\mathrm{O}_{2}$

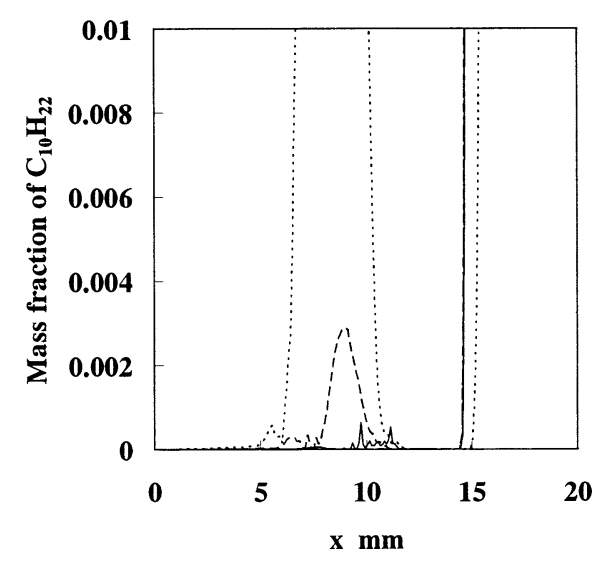

(d) Mass fraction of $\mathrm{C}_{10} \mathrm{H}_{22}$

Fig. 5 Axial profiles of time-averaged $u, T, Y_{\mathrm{O} 2}$, and $Y_{\mathrm{C} 10 \mathrm{H} 22}$ 

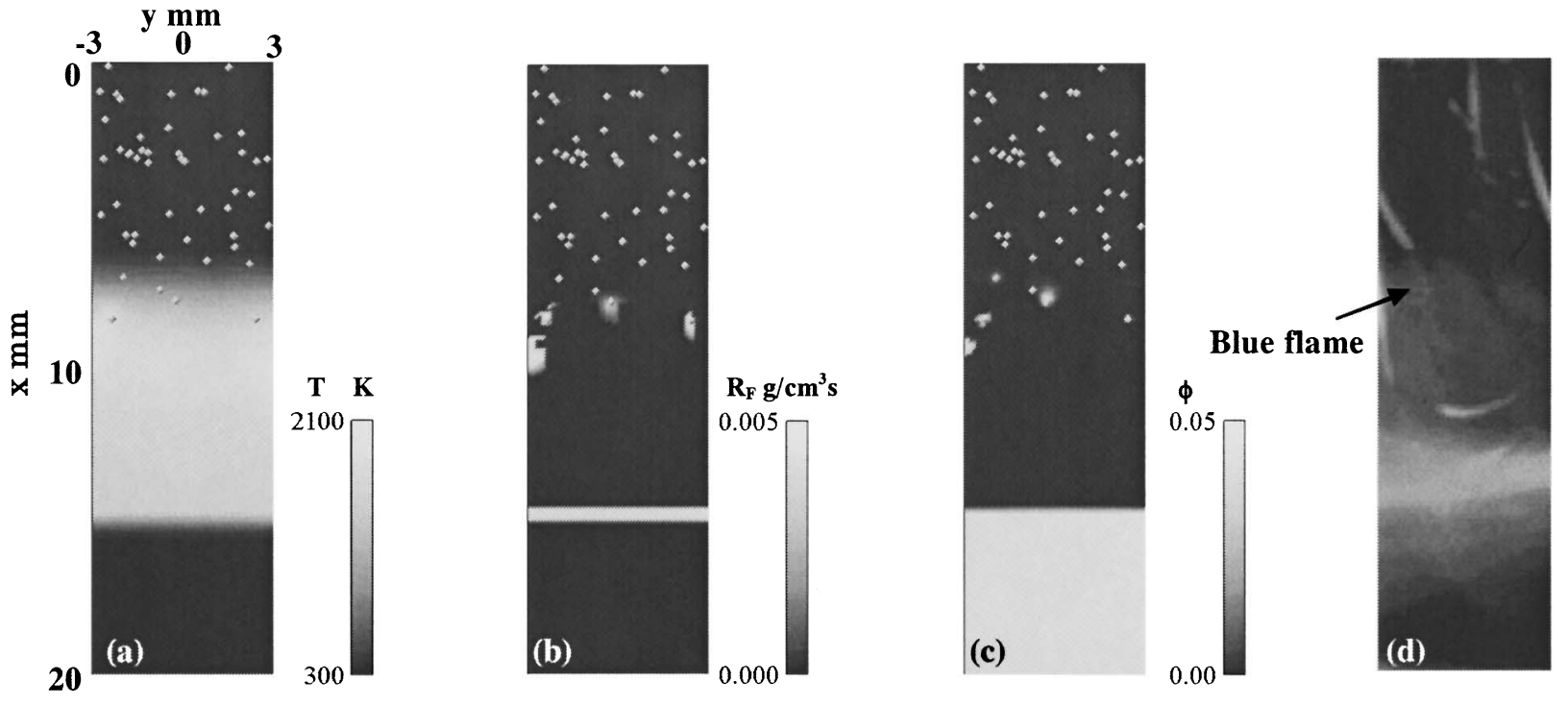

Fig. 6 Instantaneous combustion field and droplet behavior $\left(\phi_{l}=0.21, t=200 \mathrm{~ms}\right)$
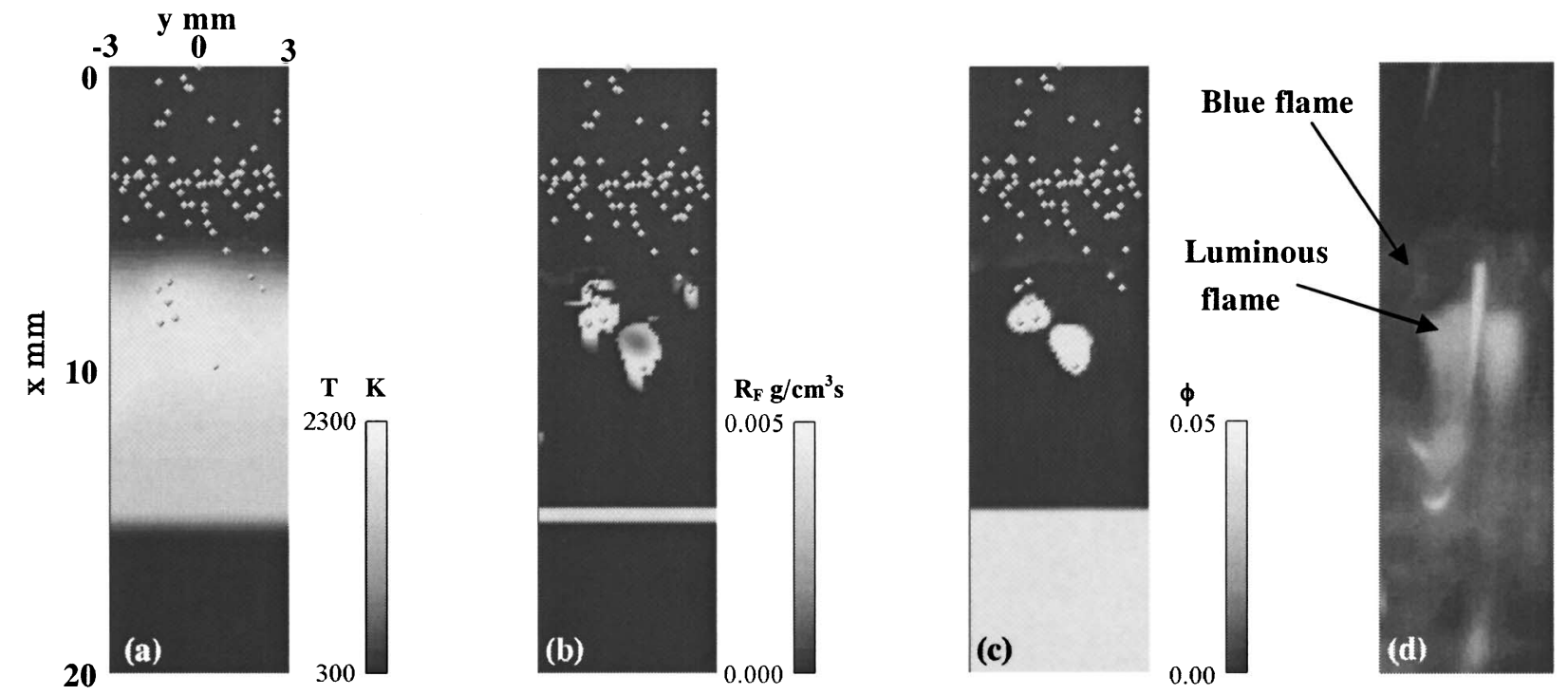

Fig. 7 Instantaneous combustion field and droplet behavior $\left(\phi_{l}=0.42, t=200 \mathrm{~ms}\right)$

When $\phi_{l}=0.42$ and 1.26, although the temperature level is almost similar, the shape of the temperature distribution near the maximum temperature is different. In Fig. 5 (c), the mass fraction of oxygen $Y_{O 2}$ in the high temperature region clearly decreases as $\phi_{l}$ increases. In Fig. 5 (d), two peaks are seen in the profile of $Y_{\mathrm{C} 10 \mathrm{H} 22}$ on the spray flow side in the cases of both $\phi_{l}=0.42$ and 1.26. The second peak is higher than the first, and a large quantity of fuel vapor is generated in the high temperature region.

As mentioned earlier, the time-averaged spray flame structures are different depending on the quantity of the supplied spray. Furthermore, we examine the instantaneous spray flame structures by comparing the numerical and experimental results.

Figures 6-8 show the instantaneous combustion fields and droplet behaviors for the three equivalence ra- tios, respectively. These results are obtained at $t=200 \mathrm{~ms}$ after the spray injection. In (a), (b), and (c), the twodimensional profiles of the gaseous phase temperature $T$, the combustion reaction rate $R_{F}$, and the gaseous phase equivalence ratio $\phi$, expressed in a gray scale, are displayed, respectively, for the areas of $x=0 \sim 20 \mathrm{~mm}$ and $y=-3 \sim 3 \mathrm{~mm}$. The droplet location is superimposed on the profiles. Photographs (d) show the flame corresponding to the numerical results.

In Fig. 6, for $\phi_{l}=0.21$, single droplets burn with an envelope flame in the high temperature region (Fig. 6(a) and (b)). This corresponds to the blue flame in the photograph shown in Fig. 6(d). For a small amount of supplied spray $\left(\phi_{l}=0.21\right)$, a single droplet combustion mode is observed due to a small quantity of fuel vapor and sufficient oxygen, as shown in Fig. 6 (c) and Fig. 5 (c) and (d). 

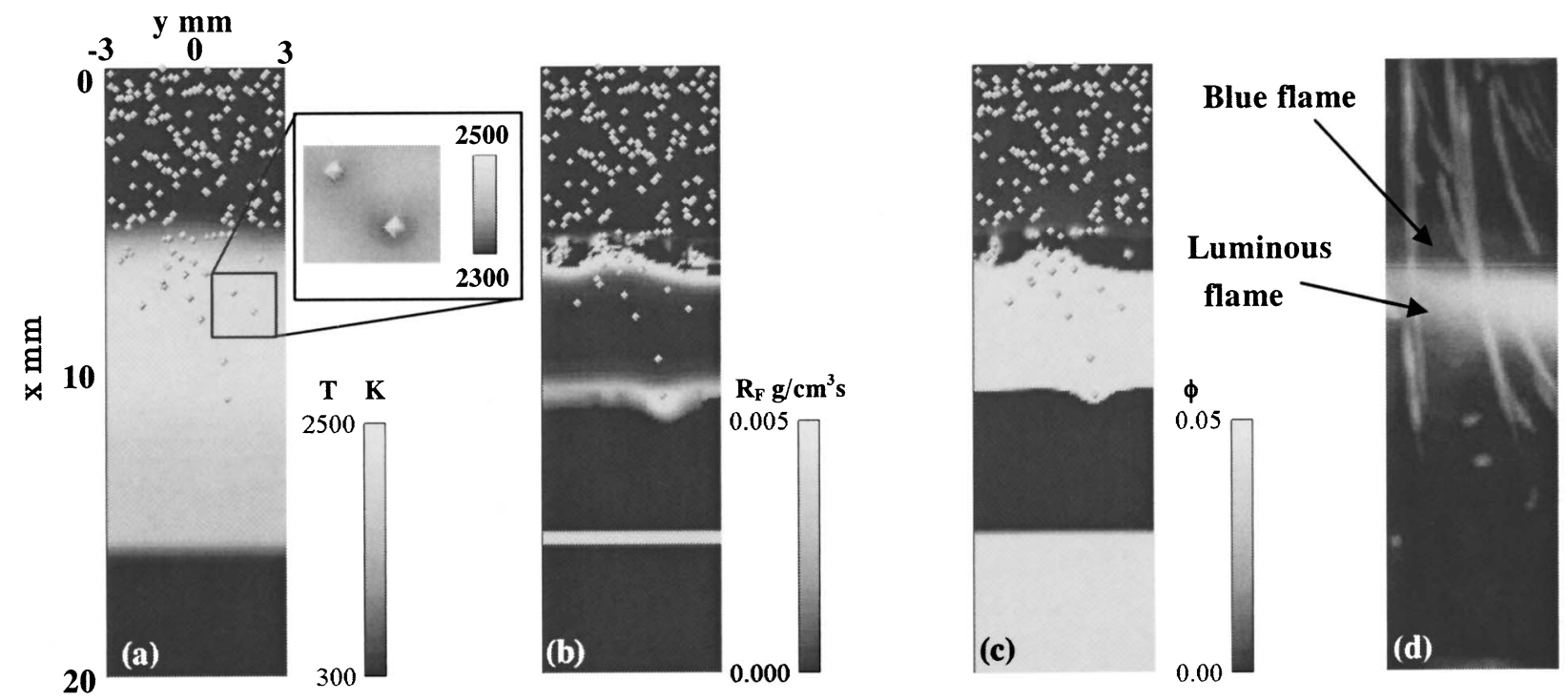

Fig. 8 Instantaneous combustion field and droplet behavior $\left(\phi_{l}=1.26, t=200 \mathrm{~ms}\right)$

Figure 7 shows the results for $\phi_{l}=0.42$. Dense fuel vapor regions are formed around the droplets burning in the diffusion combustion mode (Fig. 7 (b) and (c)), and the temperature is high around the droplets (Fig. 7 (a)). In the photograph (Fig. 7(d)), luminous flames are observed at the corresponding location. In Fig. 7 (b), a thin flame with a weak reaction rate is observed in the unburned spray side above the group flames, where the equivalence ratio $\phi$ is approximately 0.01 (Fig. 7 (c)). In this reaction zone, the prevaporization of droplets proceeds to form a premixture that burns in a premixed combustion mode. This lean premixture $(\phi \approx 0.01)$ is not flammable at an ambient temperature. In this case, however, the heat flux from the high temperature region of the diffusion flame of the droplets sustains combustion of the lean premixture. In the corresponding photograph (Fig. 7 (d)), a blue flame is observed in the upper region of the luminous flame.

Figure 8 shows the results for $\phi_{l}=1.26$. A dense fuel vapor region is formed around the droplets in the high temperature region, and burns as one group flame in the diffusion combustion mode (Fig. 8 (a) and (c)). This diffusion type group flame corresponds to the luminous flame region in the corresponding photograph (Fig. 8 (d)). As observed in Fig. 8 (b), no combustion reaction occurs inside the dense fuel vapor region because of lack of oxygen, and the gaseous temperature near the droplets is lower than that in the surroundings due to the vaporization of droplets. This decrease in the gaseous temperature is also found in Fig. 5 (b) for the time-averaged quantity. The results for $\phi_{l}=1.26$ also show that the premixed combustion region of prevaporized fuel exists in the unburned spray side above the group flame (see Fig. 8 (b) and (c)).

The abovementioned results show that the spray flame structure varies from single droplet combustion to group combustion as $\phi_{l}$ increases and the internal struc- ture of the group flame changes depending on the amount of supplied fuel spray.

\section{Conclusions}

The time-dependent combustion processes of an ndecane polydisperse spray entering a gaseous flat flame stabilized in a laminar counterflow were experimentally and numerically studied. The present numerical simulations predicted the combustion behavior obtained by the experiments. The numerical results were consistent with the typical flame structures observed by the experiment. It was found that single droplet combustion occurred in the case of a small amount of supplied fuel spray, and that double flames of lean premixed combustion of prevaporized fuel and diffusion combustion of droplet groups were observed on the spray flow side in case of a large amount of supplied fuel spray. Although this lean premixture was not flammable at an ambient temperature, the heat flux from the high temperature region of the diffusion flame of the droplets sustained the combustion of a very lean premixture. Inside the group flame, no combustion reaction occurred because of the lack of oxygen, and the gaseous temperature decreased owing to the droplet evaporation.

\section{References}

( 1 ) Chiu, H.H., Kim, H.Y. and Croke, E.J., Internal Group Combustion of Liquid Droplets, Proceedings of Combustion Institute, No.19 (1982), pp.971-980.

(2 ) Akamatsu, F., Mizutani, Y., Katsuki, M., Tsushima, S. and Cho, Y.D., Measurement of the Local Group Combustion Number of Droplet Clusters in a Premixed Spray Stream, Proceedings of Combustion Institute, No.26 (1996), pp.1723-1729.

( 3 ) Tsushima, S., Saitoh, H., Akamatsu, F. and Katsuki, M., Observation of Combustion Characteristics of Droplet Clusters in a Premixed-Spray Flame by Simul- 
taneous Monitoring of Planar Spray Images and Local Chemiluminescence, Proceedings of Combustion Institute, No.27 (1998), pp.1967-1974.

(4) Wakabayashi, T., Mizutani, Y., Katsuki, M. and Akamatsu, F., Observations of the Structure of a Spray Flame in 2-D Counterflow Burner, 36th AIAA Aerospace Sciences Meeting, (1998-2001), Paper No.98-0720.

( 5 ) Saitoh, H., Tsushima, S., Negoro, M., Akamatsu, F. and Katsuki, M., Observation of Flame Propagation in a Premixed-Spray Stagnation Flow, Proceedings of 10th International Symposium Appl. of Laser Tech. to Fluid Mech., (2000), p.30.3.

( 6 ) Greenberg, J.B., Albagli, D. and Tambour, Y., An Opposed Jet Quasi-Monodisperse Spray Diffusion Flame, Combustion Science and Technology, No.50 (1986), pp.255-270.

( 7 ) Continillo, G. and Sirignano, W.A., Counterflow Spray Combustion Modeling, Combustion and Flame, No.81 (1990), pp.325-340.

( 8 ) Li, S.C., Libby, P.A. and Williams, F.A., Spray Structure in Counterflowing Streams with and without a Flame, Combustion and Flame, No.94 (1993), pp.161177.

(9) Darabiha, N., Lacas, F., Rolon, J.C. and Candel, S., Laminar Counterflow Spray Diffusion Flames: A Comparison between Experimental Results and Complex Chemistry Calculations, Combustion and Flame, No.95 (1993), pp.261-275.

(10) Greenberg, J.B. and Sarig, N., Coupled Evaporation and Transport Effects in Counterflow Spray Diffusion Flames, Combustion Science and Technology, No.92 (1993), pp.1-33.

(11) Gomez, A. and Chen, G., Charge-Induced Secondary Atomization in Diffusion Flames of Electrostatic Sprays, Combustion Science and Technology, No.96 (1994), pp.47-59.

(12) Li, S.C., Spray Stagnation Flames, Progress in Energy and Combustion Science, No.23 (1997), pp.303-347.

(13) Gutheil, E. and Sirignano, W.A., Counterflow Spray Combustion Modeling with Detailed Transport and Detailed Chemistry, Combustion and Flame, No.113 (1998), pp.92-105.

(14) Akamatsu, F., Saitoh, H. and Katsuki, M., Numerical
Study on Combustion Characteristics of Spray Flat Flames, Proceedings of 4th KSME/JSME Thermal Engineering Conference, No.1 (2000), pp.259-264.

(15) Akamatsu, F., Saitoh, H. and Katsuki, M., Combustion Mechanisms of Spray Flat Flames Stabilized in a Laminar Counterflow, Proceedings of 3rd Asia-Pacific Conference on Combustion, (2001), pp.515-518.

(16) Kee, R.J., Rupley, F.M. and Miller, J.A., Chemkin-II: A Fortran Chemical Kinetics Package for the Analysis of Gas Phase Chemical Kinetics, SANDIA Report SAND89-8009B, (1989).

(17) Kee, R.J., Dixon-Lewis, G., Warnatz, J., Coltrin, M.E. and Miller, J.A., A Fortran Computer Code Package for the Evaluation of Gas-Phase, Multicomponent Transport Properties, SANDIA Report, SAND868246, (1986).

(18) Reid, R.C., Prausnitz, J.M. and Sherwood, T.K., The Properties of Gases and Liquids, Third Edition, (1977), McGraw-Hill, New York.

(19) Patankar, S.V., Numerical Heat Transfer and Fluid Flow, (1980), McGraw-Hill, New York.

(20) Crowe, C.T., Sharma, M.P. and Stock, D.E., The Particle-Source-in Cell (PSI-CELL) Model for GasDroplet Flows, ASME Journal of Fluids Engineering, No.99 (1977), pp.325-332.

(21) Kurose, R., Makino, H., Komori, S., Nakamura, M., Akamatsu, F. and Katsuki, M., Effects of Outflow from the Surface of a Sphere on Drag, Shear Lift, and Scalar Diffusion, Physics of Fluids, No.15 (2003), pp.23382351.

(22) Miller, R.S. and Bellan, J., Direct Numerical Simulation of a Confined Three-Dimensional Gas Mixing Layer with One Evaporation Hydrocarbon-DropletLaden Stream, Journal of Fluid Mechanics, No.384 (1999), pp.293-338.

(23) Miller, R.S., Harstad, K. and Bellan, J., Evaluation of Equilibrium and Non-Equilibrium Evaporation Models for Many-Droplet Gas-Liquid Flow Simulations, International Journal of Multiphase Flow, No.24 (1998), pp.1025-1055.

(24) Westbrook, C.K. and Dryer, F.L., Chemical Kinetic Modeling of Hydrocarbon Combustion, Progress in Energy and Combustion Science, No.10 (1984), pp.157. 